ARTÍCULO

\title{
Biodiversidad y Obras de Conservación de Agua y Suelo (OCAS) Forestadas con Especies Vegetales en Ecosistemas Áridos de la Región de Coquimbo
}

\author{
Silva, Sergio I. ${ }^{1 *}$; Gacitúa, Sandra ${ }^{1}$; Hernández, José ${ }^{1}$; Montenegro, Jaime ${ }^{1}$; Jiménez, Ismael ${ }^{1}$ \& Silva- \\ Aranguiz, Enrique? ${ }^{2}$. \\ ${ }^{1}$ Instituto Forestal Sede Diaguita, Chile. ssilva@infor.cl \\ ${ }^{2}$ CAPES-UC \\ *Autor para correspondencia.
}

\section{RESUMEN}

Con el objetivo de evaluar el efecto del uso de Obras de Conservación de Agua y Suelo (OCAS) sobre la biodiversidad en ecosistemas áridos de la Región de Coquimbo, se estudió la componente biodiversidad vegetal y fauna en cuatro áreas: 1) Con OCAS establecida en el año 1999, 2) Ambiente natural sin intervención, 3) Con OCAS establecidas en 2018 y 4) Área degradada con suelo desnudo, erosionado y perturbado.

Se realizaron mediciones estacionales de biodiversidad para las estaciones calendario primavera 2019 (octubre), verano 2020 (enero), otoño 2020 (abril), invierno 2020 (julio), primavera 2020 (octubre) y verano 2021 (enero). Durante cada medición se estableció la riqueza/abundancia de artrópodos, reptiles, aves y mamíferos. En el caso de la vegetación, se determinó la riqueza florística y cobertura vegetal.

Los resultados muestran que el área con OCAS establecidas hace 23 años y el área con ambiente natural sin intervención presentan mayor biodiversidad que los otros dos sitios evaluados (OCAS establecidas hace 3 años y área degradada sin OCAS). De esta manera, las OCAS con vegetación junto con acumular agua y conservar suelo, contribuirían a la osificación de estas áreas degradadas, traduciéndose en una recuperación importante de la biodiversidad de aves y artrópodos presentes, permitiendo concomitantemente disminuir la fragmentación y permitiendo la conectividad biológica (corredores biológicos) con otras áreas naturales de la Región.

Palabras clave: Obras de Conservación de Agua y Suelos (OCAS), Biodiversidad, Oasificación.

\section{SUMMARY}

In order to evaluate water and soils conservation practices effect on biodiversity in arid ecosystems in Coquimbo Region, Chile, the plant and animal biodiversity component was studied in four areas: 1) With OCAS established in the year 1999, 2) Natural environment without OCAS intervention, 3) With OCAS established in 2018 and 4) Degraded area.

Seasonal measurements of biodiversity were made for the calendar seasons spring 2019 (October), summer 2020 (January), autumn 2020 (April), winter 2020 (July), spring 2020 (October) and summer 2021 (January). The richness/abundance of arthropods, reptile, birds and mammals were evaluated during each season. The floristic richness and plant cover were determined in the case of vegetation.

The results show that the area with OCAS established 23 years ago and natural area without intervention shows greater biodiversity than the two other evaluated sites (OCAS established 3 years ago and degraded area without OCAS). In this way, OCAS with vegetation, water accumulation and soil conservation, would contribute to the ossification of these degraded areas, obtaining an important recovery of the biodiversity of birds and arthropods, and also manages to reduce fragmentation and allowing biological connectivity (biological corridors) with other natural areas of the region.

Key words: Soils and Water Conservation, biodiversity, oasification. 


\section{INTRODUCCIÓN}

La región de Coquimbo, de acuerdo a sus características fitogeográficas y climáticas, se encuentra clasificada mayoritariamente como una zona árida y con altos niveles de degradación (CIREN, 2012; Gore Coquimbo, 2017; DGA, 2021), cuya afectación cubre un 96\% del territorio en condición de riesgo de desertificación grave y media y solo un 4\% con riesgo de desertificación leve (CIREN, 2012).

Las actividades antrópicas están sindicadas como las mayores responsables del estado actual de los suelos en la región; esta condición se originó en un principio por una fuerte explotación de leña y producción de carbón y posteriormente, durante los últimos treinta años, debido al masivo cultivo de grandes superficies de trigo y cereales (CIREN, 2010; CONAF, 2021).

Las pérdidas de suelos pueden llegar a las 20 (t/ha/año) en sectores montañosos, mientras que en sectores de serranía ellas alcanzarían una cifra entre 2 y 10 (t/ha/año). En las zonas de cultivo en laderas las pérdidas podrían alcanzar a 50 (t/ha/año) lo que conduce a una fuerte degradación del suelo en un período de 15 a 20 años (Jorquera, 2001; AGRIMED, 2017).

Las comunas con mayor proporción de suelos erosionados bajo alguna de sus categorías son CanelaIllapel $(93,8 \%)$, la Higuera $(94,8 \%)$ y Punitaqui $(93,8 \%)$. Con respecto a los suelos de las comunas de Canela-Illapel, se encuentran entre los más antiguos del país, llegando a constituir duripanes que limitan la penetración de las raíces, con pérdida de material orgánico en su estructura superficial, generado en gran medida por el mal manejo representado por el sobrepastoreo, extracción de leña, aradura de suelos en pendientes y monocultivo de cereales (Morales et al., 2016; CIREN, 2010, 2012; Gore Coquimbo, 2010, 2016).

En cuanto al recurso hídrico, la región presenta 3,9 millones de hectáreas afectadas por sequía grave, siendo la población rural la más vulnerable a sus efectos (CONAF, 2016). Esto significa que la disponibilidad de agua no alcanza a satisfacer la demanda para sus distintos usos, cuyas causas son producto del incremento de la erosión de los suelos, la pérdida de infiltración de agua en napas freáticas, el uso inadecuado del agua y la alta competencia que existe por la utilización de este recurso en el marco de una legislación deficiente que permite su sobreexplotación por intereses económicos foráneos (Larraín et al., 2010; Gore Coquimbo, 2017).

La vulnerabilidad de los ecosistemas y la biodiversidad de las zonas rurales de la región de Coquimbo se ven empeoradas aún más por el fenómeno del cambio climático, producto de la intensificación de las alteraciones en los patrones climáticos a una velocidad superior a la capacidad adaptativa de los organismos vivos.

La fragmentación de los ecosistemas es considerada como una de las principales causantes de grandes cambios en el ambiente físico-biótico, en donde composición, estructura y función original de un ecosistema se han alterado (por ejemplo, la pérdida en la conectividad, la creación de bordes sobre el hábitat o el aislamiento de fragmentos), provocando dinámicas muy diferentes sobre las poblaciones biológicas que allí habitan (Bustamante y Grez, 1995; Bennett, 1998, 2003; Vásquez y Simonetti, 1999, Awade y Metzger, 2008).

Estos factores modifican la composición y abundancia de las especies de un ecosistema pudiendo facilitar o impedir el mantenimiento de algunas de ellas, por lo que distintos grupos de organismos (ej. insectos, reptiles, aves y mamíferos) han sido considerados útiles para evaluar la calidad, recuperación del hábitat y cambios en las interacciones tróficas (Sarmiento et al., 2002; Pizarro et al., 2004; Arim et al. 2007, McRae et al., 2012).

En la lucha contra la desertificación en zonas áridas se ha actuado habitualmente con técnicas tradicionales de repoblación vegetal. Sin embargo, en estos lugares es preciso diseñar la restauración de la vegetación con criterios de economía del agua. Hay que apostar por invertir el proceso de desertificación mediante su opuesto, es decir, mediante la oasificación (Pizarro et al., 2004; Martínez de Azagra et al., 2006; Mongil y Martínez de Azagra, 2006, Perret et al., 2011). Se trata de crear pequeñas estructuras de tierra que frenen la escorrentía que fluye por la ladera (OCAS), consiguiendo no solo un microclima más húmedo en el suelo de la zona de plantación, sino mejores condiciones edáficas, factores que ayudan a la instalación de vegetación nativa, que revierta el proceso de degradación y a su vez recupere servicios ecosistémicos, entre ellos, la biodiversidad (Hernández et al., 2020). 
Desde esta perspectiva, los enfoques multipropósito que involucren sistemas agroforestales, OCAS y elaboración de modelos predictivos pueden apoyar la toma de decisiones sobre la priorización de áreas dentro de los paisajes en donde la oasificación mediante la implementación de OCAS traerá no solo la conservación de agua y suelo, sino que muy probablemente altos beneficios para la biodiversidad (Rappaport et al., 2015 Tambosi et al., 2014; Pizarro et al., 2004; Gacitúa et al., 2020).

El Instituto Forestal desde hace 25 años aproximadamente ha establecido OCAS en la zona centro norte de Chile, obras que permiten fundamentalmente cosechar aguas lluvias, agua que se acumula en el suelo e infiltra hacia las napas subterráneas, constituyéndose en un aporte hídrico para la vegetación y también para la sociedad.

Esta obras contribuyen a combatir la degradación de los suelos y el deterioro de los ecosistemas, proceso que, más allá de la conservación de agua y suelo, constituye una oasificación de estos lugares, recuperando también biodiversidad, ya que, tal como indicado previamente, con la oasificación se aumenta la resiliencia de la vegetación y biodiversidad, es decir, se incrementa la probabilidad de recuperar los ecosistemas degradados (Perret et al., 2011; Labra et al., 2018; González et al., 2020; Hernández et al., 2020).

\section{OBJETIVOS}

El objetivo del estudio fue evaluar el efecto del uso de Obras de Conservación de Agua y Suelo (OCAS) sobre la biodiversidad en ecosistemas áridos de la región de Coquimbo.

\section{MATERIAL Y MÉTODO}

\section{Área de Estudio}

Las áreas de estudio están ubicadas en la región de Coquimbo, provincias del Limarí y Choapa, específicamente en las comunas de Canela e Illapel, que corresponden a una estepa con influencia marina con régimen de humedad árido (AGRIMED, 2017).

Estas áreas se ubican en predios de las Comunidades Agrícolas Carquindaño y Tunga Norte, donde el Instituto Forestal (INFOR) desde hace 25 años y en colaboración con ellas, ha establecido una red de unidades pilotos con OCAS para la recuperación de bienes y servicios ecosistémicos (Figura 1).

En términos vegetacionales se ubican en la formación Matorral Desértico, específicamente en los bordes transicionales entre los pisos del Matorral Desértico Mediterráneo Costero de Bahia ambrosioides y Puya chilensis; y el piso Matorral Desértico Mediterráneo Interior de Flourensia thurifera y Colliguaja odorifera (Gajardo, 1994; Luebert y Pliscoff, 2006).

Históricamente estas áreas han estado sometidas a intensas perturbaciones de origen antrópico (ej. sobrepastoreo, extracción de leña, uso intensivo de la tierra) hecho que se traduce en fuertes procesos erosivos que han modelado su actual relieve.

Climáticamente se insertan dentro del dominio del clima de Estepa Templado Marginal, con precipitaciones con promedio anual que no supera los $100 \mathrm{~mm}$. Las temperaturas medias anuales varían entre $12^{\circ}$ y $16^{\circ}$ C siendo su oscilación entre el mes más frío y el mes más cálido de alrededor de $8^{\circ}$ a $10^{\circ} \mathrm{C}$.

Desde un punto vista hidrográfico, estas áreas se abastecen de agua a través de una densa red de drenaje de tipo estacional con quebradas que se activan en función de las precipitaciones intensas y concentradas durante los meses de invierno (junio-agosto; Jorquera, 2001; Flores et al., 2010; AGRIMED, 2017). 


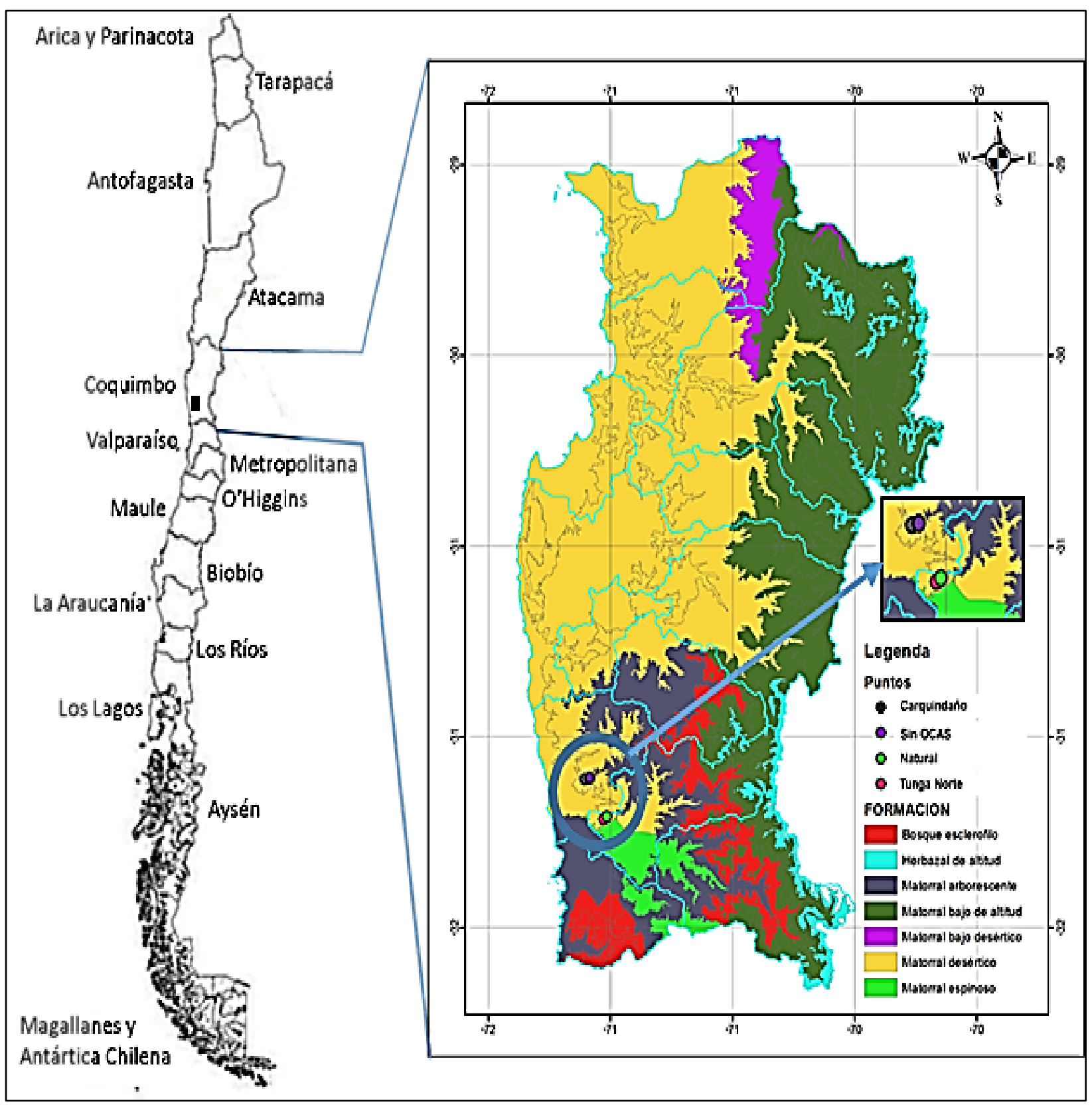

(Fuente: elaboración propia usando formciones de Luebert y Pliscoff, 2006)

Figura 1. Mapa de la Región de Coquimbo Indicando las Áreas de Estudio

Para el desarrollo del estudio fueron seleccionadas cuatro áreas en función del tiempo cronológico de intervención, descritas en el Cuadro 1. 
Cuadro 1. Descripción y Coordenadas Geográficas de las Áreas de Estudio.

\begin{tabular}{|c|c|c|c|c|c|}
\hline \multirow{2}{*}{$\begin{array}{c}\text { Área de } \\
\text { Estudio } \\
\text { (Situaciones } \\
\text { evaluadas) }\end{array}$} & \multirow{2}{*}{ Descripción } & \multirow{2}{*}{$\begin{array}{l}\text { Tipo de OCAS } \\
\text { Establecidas }\end{array}$} & \multirow{2}{*}{$\begin{array}{l}\text { Vegetación } \\
\text { Establecida }\end{array}$} & \multicolumn{2}{|c|}{$\begin{array}{c}\text { Coordenadas } \\
\text { WGS84 }\end{array}$} \\
\hline & & & & Este & Norte \\
\hline Tunga Norte & $\begin{array}{l}\text { Con OCAS establecida en } \\
\text { el año 1999. Abarca 2,5 ha } \\
\text { delimitada por cerco anti } \\
\text { herbívoros grandes (ej. } \\
\text { cabras, caballos) }\end{array}$ & $\begin{array}{l}\text { Surco en media } \\
\text { luna, limanes y } \\
\text { zanjas de } \\
\text { infiltración }\end{array}$ & $\begin{array}{c}\text { Eucalyptus sp, } \\
\text { Prosopis chilensis, } \\
\text { Quillaja saponaria } \\
\text { Caesalpinia spinosa }\end{array}$ & 279143 & $\begin{array}{c}649785 \\
2\end{array}$ \\
\hline Natural & $\begin{array}{l}\text { Área no intervenida, con } \\
\text { ambiente natural }\end{array}$ & Sin OCAS & $\begin{array}{c}\text { Formación matorral } \\
\text { desértico }\end{array}$ & 280462 & $\begin{array}{c}649904 \\
0\end{array}$ \\
\hline Carquindaño & $\begin{array}{c}\text { Área con OCAS, } \\
\text { establecida en el año } \\
\text { 2018. Abarca } 3 \text { ha } \\
\text { delimitada por cerco anti } \\
\text { herbívoros grandes (ej. } \\
\text { cabras, caballos) }\end{array}$ & $\begin{array}{l}\text { Surco en media } \\
\text { luna, limanes y } \\
\text { zanjas de } \\
\text { infiltración }\end{array}$ & $\begin{array}{c}\text { Cordia decandra, } \\
\text { Prosopis chilensis, } \\
\text { Acacia saligna, } \\
\text { Ficus carica }\end{array}$ & 270832 & $\begin{array}{c}651442 \\
2\end{array}$ \\
\hline Sin OCAS & $\begin{array}{c}\text { Área degradada con suelo } \\
\text { desnudo y erosionado }\end{array}$ & Sin OCAS & Sin vegetación & 271034 & $\begin{array}{c}651446 \\
1 \\
\end{array}$ \\
\hline
\end{tabular}

\section{Monitoreo de Biodiversidad}

La biodiversidad presente en las distintas áreas de estudio fue monitoreada durante las estaciones calendario primavera 2019 (octubre), verano 2020 (enero), otoño 2020 (abril), invierno 2020 (julio), primavera 2020 (octubre) y verano 2021 (enero).

En cada ocasión se estableció la riqueza/abundancia de artrópodos, reptiles, aves y mamíferos; para el caso de la vegetación, la riqueza florística y cobertura se determinó en base a parcelas de inventario tanto para la el estrato arbóreo/arbustivo como para el estrato herbáceo. La metodología para evaluar cada componente en las áreas de estudio se detalla en los párrafos siguientes.

Flora: Para la caracterización de la riqueza florística y cobertura de la vegetación arbórea/arbustiva, se establecieron cinco parcelas circulares, de $8 \mathrm{~m}$ de radio, abarcando una superficie aproximada de $200 \mathrm{~m}^{2}$.

Para el caso de la cobertura herbácea se utilizó cinco parcelas de $1 \mathrm{~m}^{2}$. Las parcelas fueron distribuidas aleatoriamente dentro de cada área de estudio. Los datos obtenidos de los muestreos permiten estimar la cobertura vegetal arbórea/arbustiva y herbácea absoluta de cada parcela, definida como la proporción de la superficie del suelo cubierto por la proyección perpendicular de las especies vegetales.

Artrópodos: Fueron monitoreados mediante trampas Barber, que consisten en utilizar un vaso plástico de $100 \mathrm{cc}$ de forma cónica y de $60 \mathrm{~mm}$ de diámetro en su boca, relleno con agua y detergente para disminuir la tensión superficial del agua. Los vasos fueron enterrados, quedando su parte superior a nivel de suelo. Se instalaron 5 estaciones de muestreo equidistantes a 15 metros entre sí, cada una compuesta por 5 Barber distanciadas al menos $50 \mathrm{~cm}$ una de otra.

Se contabilizó un total de 25 vasos por área de estudio, los cuales permanecieron activos por un periodo de 48 horas. La identificación de artrópodos capturados fue a nivel de Orden.

Reptiles: Se utilizaron técnicas no invasivas basadas en la recolección de evidencias directas e indirectas. Para cada área de estudio se implementó un transecto de $50 \mathrm{~m}$ de largo y $20 \mathrm{~m}$ de ancho, muestreado por dos observadores.

La modalidad de detección fue sobre la base de avistamiento durante los transectos y evidencias indirectas (huellas, mudas, fecas y presencia de restos óseos en egagrópilas). La abundancia se estimó en base al número total de individuos observados para los transectos. 
Para la identificación de las especies de reptiles observados en el terreno se utilizaron guías de campo e información presente en la literatura especializada tales como Veloso y Navarro, 1988; Núñez y Jaksic, 1992; Mella, 2005; Pincheira y Núñez, 2005.

Aves: Los censos fueron realizados por un equipo de dos personas, provistos de binoculares. Esto aumenta la probabilidad de observar todas las aves que están presentes en el área, lo que permite una identificación segura de las especies (Reynolds et al., 1980, Blondel et al., 1981, Willson et al., 1994, Jiménez, 2000).

Los puntos de observación y escucha fueron establecidos en el centro de cada área de estudio, en todos los casos el radio de observación en cada punto fue aproximadamente de 80-100 m, lo que cubre un área aproximada de 2-3 ha. Se realizaron preferentemente durante días despejados, entre las 08:00 y 10:00 horas, durante 15 minutos.

Mamíferos: Se utilizaron técnicas de muestreo del tipo no invasivo, que incluyen el uso de evidencias indirectas como fecas, huellas, pelos y madrigueras. Adicionalmente se realizaron observaciones sistemáticas a través de un único transecto de 50 metros de largo y $20 \mathrm{~m}$ de ancho y un punto de observación.

En cada punto se registraron los mamíferos presentes en un radio de $100 \mathrm{~m}$, utilizando binoculares Bushnell (10x50) por 10 minutos. Los recuentos fueron efectuados por dos observadores, entre las 8:00 y las 18:00 horas.

Para la identificación de las distintas especies observadas y descritas se usó guías e información especializada, principalmente la contenida en Muñoz-Pedreros y Yáñez (2000) e Iriarte (2008).

\section{Análisis de Variables de Biodiversidad}

Solo para el caso de las aves se calculó el índice de diversidad Alfa (Índice de Shannon-Weaver, Magurran, 1988; 2004), que hace referencia a la diversidad local dentro de una comunidad y el índice de diversidad Beta (Índice de Jaccard; Magurran, 1988; 2004), la cual representa el cambio en la diversidad entre dos 0 más comunidades (Martínez et al., 2009; Armendaño y González, 2010).

Estos índices son sensibles a n muestrales bajos $(<20)$, lo que se traduce en sobreestimación de estos (Magurran, 1988; 2004).

-Índice de Shannon-Wiener:

Donde:

$$
H^{\prime}=-\sum_{i=1}^{S} p_{i} \ln p_{i}
$$

$S=$ Número de especies (riqueza de especies).

$\mathrm{p}_{i}=$ Proporción de individuos de la especie $i$ respecto al total de individuos (es decir, abundancia relativa de la especie $i): n_{i} / N$.

$n_{i}=$ Número de individuos de la especie $i$.

$N=$ Número de todos los individuos de todas las especies.

El índice incluye la cantidad de especies presentes en el área de estudio (riqueza de especies) y la cantidad relativa de individuos de cada una de esas especies (abundancia).

-Índice de Jaccard:

$$
I_{j}=\frac{c}{a+b-c}
$$

Donde:

$\mathrm{a}=$ Número de especies presentes en el sitio $\mathrm{A}$.

$b=$ Número de especies presentes en el sitio $B$.

c= Número de especies presentes en ambos sitios A y B.

Las comparaciones estadísticas de abundancia y riqueza de especies entre estaciones calendario se realizó mediante análisis de la varianza (ANDEVA), previa verificación que los datos cumplieran con los 
requisitos de normalidad y homocedasticidad de varianzas; para comparaciones a posteriori se utilizó la prueba de Tukey $(\alpha=0.05)$.

\section{RESULTADOS}

\section{Riqueza Florística}

Se identificó un total de 24 taxa de plantas vasculares, 20 especies para el área Natural, 11 para Tunga Norte, 6 para Carquindaño y 4 para Sin OCAS. En cuanto a la cobertura arbórea/arbustiva los valores en orden decente fueron: Natural $(84 \%)>$ Tunga Norte $(56 \%)>$ Carquindaño $(3,4 \%)>$ Sin OCAS $(2,2 \%)$. Los valores de cobertura arbórea/arbustiva fueron significativamente diferentes $\left(F_{(3,16)}=32,30 ; P<0,001\right)$ y la prueba de Tukey permitió diferenciar dos grupos, el primero conformado por Tunga Norte/ Natural y otro por Carquindaño y Sin OCAS ( $P<0.05$; Nivel de confianza 95\%).

Para el caso de la cobertura herbácea, el patrón observado fue: Tunga Norte (86\%) > Natural (33\%) > Carquindaño $(12 \%)>$ Sin OCAS $(0,4 \%)$ (Cuadro 2). Los valores de cobertura registrados son significativamente diferentes $\left(F_{(3,16)}=32,30 ; P<0,001\right)$ y la prueba de Tukey diferenció tres grupos, uno conformado por Tunga Norte, otro por Natural y por último Carquindaño/Sin OCAS $(P<0.05$; Nivel de confianza 95\%).

Cuadro 2. Especies de Flora Presentes Según Área de Estudio.

\begin{tabular}{|c|c|c|c|c|c|c|}
\hline $\mathbf{N}^{\circ}$ & Especie & Nombre común & Tunga Norte & Natural & Carquindaño & Sin OCAS \\
\hline 1 & Acacia caven & espino & $x$ & $x$ & $x$ & \\
\hline 2 & Acacia saligna & acacia & $x$ & $x$ & $x$ & \\
\hline 3 & Adesmia bedwellii & varilla brava & & $x$ & & \\
\hline 4 & Aristeguietia salvia & salvia & & $x$ & & \\
\hline 5 & Bromus sp. & bromus & $x$ & $\mathrm{x}$ & & $x$ \\
\hline 6 & Caesalpinia spinosa & tara & $\mathrm{x}$ & & & \\
\hline 7 & Cestrum parqui & palqui negro & & $x$ & & \\
\hline 8 & Cistanthe grandiflora & pata de guanaco & & $\mathrm{x}$ & & \\
\hline 9 & Cordia decandra & carbonillo & & $\mathrm{x}$ & $x$ & \\
\hline 10 & Echinopsis chiloensis & quisco & & $x$ & $x$ & \\
\hline 11 & Eucalyptus cladocalyx & eucalipto & $x$ & & & \\
\hline 12 & Gutierrezia resinosa & pichanilla & $\mathrm{x}$ & $x$ & & \\
\hline 13 & Helenium aromaticum & manzanilla & $\mathrm{x}$ & $\mathrm{x}$ & & \\
\hline 14 & Heliotropium stenophyllum & palo negro & & & & \\
\hline 15 & Lobelia polyphylla & tabaco del diablo & & $x$ & & \\
\hline 16 & Porlieria chilensis & guayacán & & $\mathrm{x}$ & & \\
\hline 17 & Prosopis chilensis & algarrobo & $x$ & $x$ & $x$ & \\
\hline 18 & Proustia ilicifolia & huañil & & $\mathrm{x}$ & & \\
\hline 19 & Quillaja saponaria & quilay & $x$ & & & \\
\hline 20 & Schinus latifolius & molle & & $x$ & & \\
\hline 21 & Schinus polygamus & huingán & & $x$ & & $x$ \\
\hline 22 & Senecio sp. & senecio & $\mathrm{x}$ & $\mathrm{x}$ & & $\mathrm{x}$ \\
\hline 23 & Senna cumingii & alcaparra & & $\mathrm{x}$ & $x$ & $\mathrm{x}$ \\
\hline 24 & Stipa sp. & gramínea & $\mathrm{x}$ & $\mathrm{x}$ & & \\
\hline \multicolumn{3}{|c|}{ Cobertura arbórea/arbustiva (\% } & 56,0 & 84,0 & 3,4 & 2,2 \\
\hline \multicolumn{3}{|c|}{ Cobertura herbáceas (\%) } & 86,0 & 33,0 & 12,0 & 0,4 \\
\hline \multicolumn{3}{|c|}{ Riqueza florística } & 11 & 20 & 6 & 4 \\
\hline
\end{tabular}

\section{Composición de Especies Fauna (artrópodos, reptiles, aves, mamíferos)}

El análisis por especie (Cuadro 3), independiente del área de estudio, indica que durante el periodo de monitoreo se registró en total 29 aves paserinas, 2 falconiformes (tiuque y cernícalo) y 1 Strigiforme (chuncho). Dentro del grupo de las aves paserinas destaca la abundancia del chincol, diuca, picaflor chico, yal y cachudito (10, 9, 9, 7 y 7 individuos, respectivamente). 
Los artrópodos registrados representan 7 ordenes (Scorpiones, Araneae, Orthoptera, Coleoptera, Hymenoptera, Diptera y Lepidoptera), le siguen los mamíferos con dos especies (Phyllotis darwini y Spalacopus cyanus); y por último se registró sólo un reptil (Liolaemus lemniscatus). Los mayores valores observados de artrópodos se registraron para el área Natural (sin intervención) y en Tunga Norte (OCAS de 23 años).

Desde el punto de vista estacional, los mayores valores tanto de abundancia como de riqueza de especies correspondieron a la estación primavera, periodo en que la mayoría de las especies están culminando su etapa reproductiva, por lo tanto, hay un reclutamiento de nuevos individuos a la comunidad existente en el área (Cuadro 4).

La riqueza de especies de artrópodos para el área Tunga Norte, es decir, con OCAS de 23 años y área Natural no intervenida, fueron significativamente mayor que lo detectado en las áreas Carquindaño y Sin OCAS, con máximos estacionales de 7 Órdenes para ambos casos.

En cambio, en Carquindaño y área degradada sin OCAS, los valores máximos estacionales observados fueron de 3 Órdenes para ambos casos $\left(F_{(3,20)}=25,53 ; P<0,001\right)$.

En el caso de las aves, se observa un patrón de diversidad similar al de artrópodos, con máximos valores estacionales de diversidad de aves en áreas de estudio Natural y Tunga Norte, en cambio, para las áreas Carquindaño y $\operatorname{Sin}$ OCAS se observan los menores registros, con 21,16, 5 y 2 especies, respectivamente, $F_{(3,20)}=38,85 ; P<0,001$ (Cuadro 3, Figura 2).

En Tunga Norte se detectó al reptil Liolaemus lemniscatus y a los roedores Phyllotis darwini y Spalacopus cyanus, este último un mamífero fosorial que prefiere hábitat abiertos bien drenados, de suelo blando y con gran cubierta vegetal de matorrales y praderas, mayor al 60\% (Root-Bernsteina y Jaksic, 2015), tal como ocurre en Tunga Norte.

Los grupos taxonómicos más representados fueron las aves y artrópodos, alcanzando sus máximos de biodiversidad durante primavera y verano, seguido por los reptiles y mamíferos (Cuadro 4, Figura 2).

Para las áreas Carquindaño y Sin OCAS no se registró presencia de reptiles ni mamíferos.

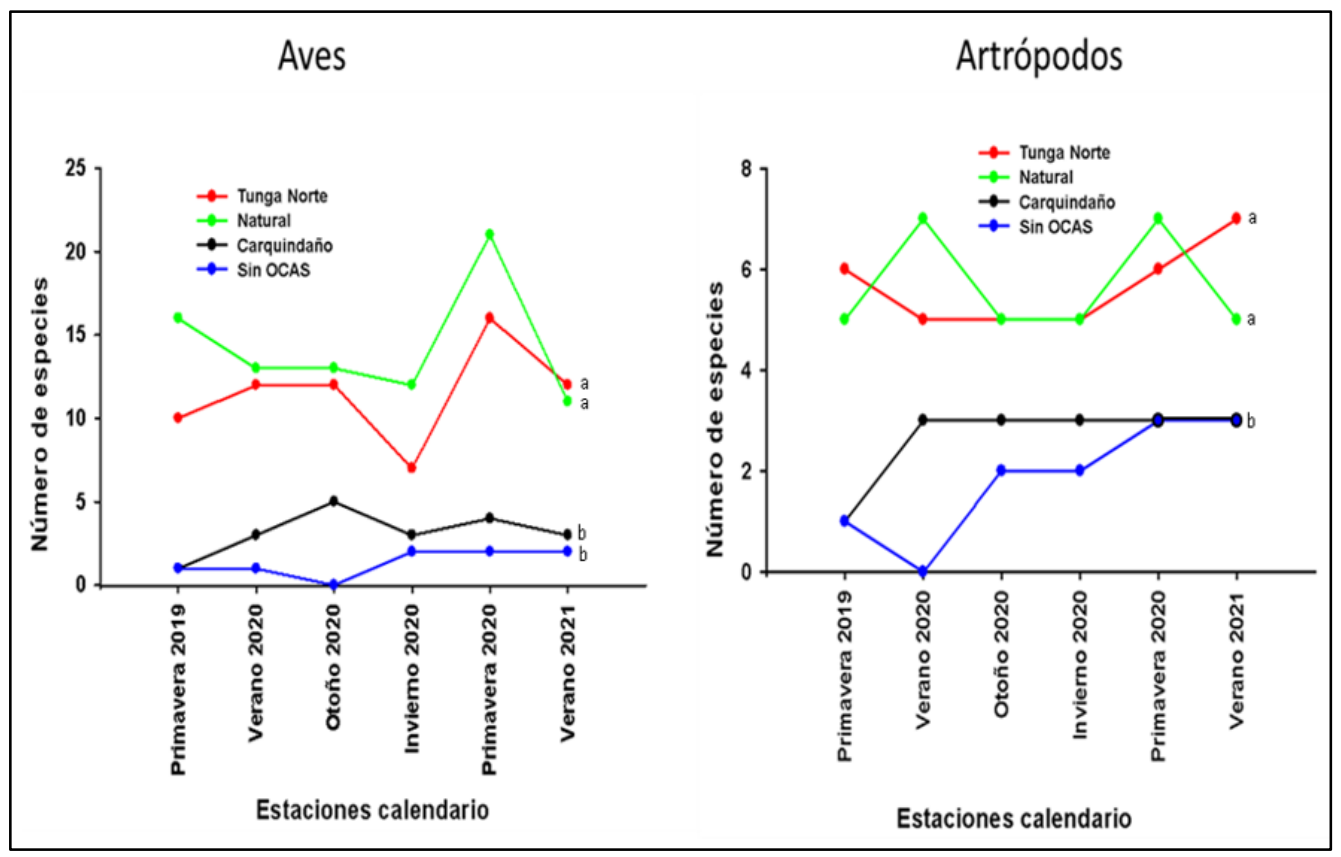

Figura 2. Riqueza de Especies para los Dos Grupos Taxonómicos Más Representados en las Áreasde Estudio. (Letras distintas indican diferencias significativas ( $p<0.05$; prueba de Tukey; nivel de confianza 95\%) 
Cuadro 3. Número de Especies/Individuos de Aves Registradas, Según Área Evaluada (Origen: $\mathrm{N}=$ Nativo, En= endémico, Ex= Exótico)

\begin{tabular}{|c|c|c|c|c|c|c|c|c|c|c|c|c|c|c|c|}
\hline \multirow[b]{2}{*}{ Especie } & \multirow{2}{*}{$\begin{array}{l}\text { Nombre } \\
\text { común }\end{array}$} & \multirow[b]{2}{*}{ Familia } & \multirow[b]{2}{*}{ Origen } & \multicolumn{4}{|c|}{ Primavera 2019} & \multicolumn{4}{|c|}{ Verano 2020} & \multicolumn{4}{|c|}{ Otoño 2020} \\
\hline & & & & $\begin{array}{l}\text { Tunga } \\
\text { Norte }\end{array}$ & Natural & $\begin{array}{l}\text { Carquin- } \\
\text { daño }\end{array}$ & $\begin{array}{c}\text { Sin } \\
\text { OCAS }\end{array}$ & $\begin{array}{l}\text { Tunga } \\
\text { Norte }\end{array}$ & Natural & $\begin{array}{l}\text { Carquin- } \\
\text { daño }\end{array}$ & $\begin{array}{c}\text { Sin } \\
\text { OCAS }\end{array}$ & $\begin{array}{l}\text { Tunga } \\
\text { Norte }\end{array}$ & Natural & $\begin{array}{l}\text { Carquin- } \\
\text { daño }\end{array}$ & $\begin{array}{c}\text { Sin } \\
\text { OCAS }\end{array}$ \\
\hline $\begin{array}{l}\text { Anairetes } \\
\text { parulus }\end{array}$ & cachudito & Tyrannidae & $\mathrm{N}$ & 2 & 7 & & & 1 & 4 & & & 3 & & & \\
\hline $\begin{array}{l}\text { Pseudasthenes } \\
\text { humicola }\end{array}$ & canastero & Furnariidae & En & & 2 & & & 1 & 1 & & & & & & \\
\hline $\begin{array}{l}\text { Asthenes } \\
\text { modesta }\end{array}$ & $\begin{array}{l}\text { canastero } \\
\text { chico }\end{array}$ & Furnariidae & $\mathrm{N}$ & & & & & & & & & & 1 & & \\
\hline $\begin{array}{l}\text { Dryobates } \\
\text { lignarius }\end{array}$ & carpinterito & Picidae & $\mathrm{N}$ & & & & & & & & & & 1 & & \\
\hline $\begin{array}{l}\text { Falco } \\
\text { sparverius }\end{array}$ & cernícalo & Falconidae & $\mathrm{N}$ & & & & & & 1 & & & & & & \\
\hline $\begin{array}{l}\text { Troglodytes } \\
\text { aedon }\end{array}$ & chercán & Troglodytidae & $\mathrm{N}$ & & 2 & & & & & & & & & & \\
\hline $\begin{array}{l}\text { Zonotrichia } \\
\text { capensis }\end{array}$ & chincol & Emberizidae & $\mathrm{N}$ & 10 & 10 & & & 2 & 2 & & 2 & 3 & 1 & & \\
\hline Sicalis luteola & chirihue & Thraupidae & $\mathrm{N}$ & & & & & & & & & & & & \\
\hline $\begin{array}{l}\text { Glaucidium } \\
\text { nana }\end{array}$ & chuncho & Strigidae & $\mathrm{N}$ & 1 & & & & & & & & & & & \\
\hline $\begin{array}{l}\text { Callipepla } \\
\text { californica }\end{array}$ & codorniz & Odontophoridae & Ex & & 1 & & & & & & & & & & \\
\hline Phrygilus gayi & $\begin{array}{l}\text { cometocino } \\
\text { de gay }\end{array}$ & Thraupidae & $\mathrm{N}$ & & 5 & & & & 3 & & & & & & \\
\hline Diuca diuca & diuca & Thraupidae & $\mathrm{N}$ & 9 & 3 & & & 2 & 3 & 1 & & 2 & 4 & 2 & \\
\hline Pyrope pyrope & diucón & Tyrannidae & $\mathrm{N}$ & 1 & & & & & & & & 1 & & & \\
\hline $\begin{array}{l}\text { Spinus } \\
\text { barbatus }\end{array}$ & jilguero & Fringillidae & $\mathrm{N}$ & 1 & & & & & & & & & & & \\
\hline Leistes loyca & loica & Ictiridae & $\mathrm{N}$ & 2 & 1 & & & 2 & & & & 3 & 2 & & \\
\hline Agriornis lividus & mero & Tyrannidae & En & & 1 & & & & & & & & & & \\
\hline $\begin{array}{l}\text { Sephanoides } \\
\text { sephaniodes }\end{array}$ & $\begin{array}{l}\text { picaflor } \\
\text { chico }\end{array}$ & Trochillidae & $\mathrm{N}$ & & 4 & & & 4 & 9 & 1 & & 2 & & & \\
\hline Patagona gigas & $\begin{array}{l}\text { picaflor } \\
\text { grande }\end{array}$ & Trochillidae & $\mathrm{N}$ & & 1 & & & 2 & 5 & & & & & & \\
\hline Colaptes pitius & pitio & Picidae & $\mathrm{N}$ & & & & & & 1 & & & & & & \\
\hline $\begin{array}{l}\text { Scelorchilius } \\
\text { albicollis }\end{array}$ & tapaculo & Rhinocryptidae & En & & 2 & & & & & & & & 1 & & \\
\hline Mimus thenca & tenca & Mimidae & En & 4 & 5 & 1 & 1 & 5 & 3 & & & 4 & 3 & 2 & \\
\hline $\begin{array}{l}\text { Leptasthenura } \\
\text { aegithaloides }\end{array}$ & tijeral & Furnariidae & $\mathrm{N}$ & & 3 & & & & 2 & & & 1 & 2 & & \\
\hline $\begin{array}{l}\text { Milvago } \\
\text { chimango }\end{array}$ & tiuque & Falconidae & $\mathrm{N}$ & & & & & & 3 & 2 & & & & & \\
\hline
\end{tabular}




\begin{tabular}{|c|c|c|c|c|c|c|c|c|c|c|c|c|c|c|c|}
\hline \multirow[b]{2}{*}{ Especie } & \multirow{2}{*}{$\begin{array}{l}\text { Nombre } \\
\text { común }\end{array}$} & \multirow[b]{2}{*}{ Familia } & \multirow[b]{2}{*}{ Origen } & \multicolumn{4}{|c|}{ Primavera 2019} & \multicolumn{4}{|c|}{ Verano 2020} & \multicolumn{4}{|c|}{ Otoño 2020} \\
\hline & & & & $\begin{array}{l}\text { Tunga } \\
\text { Norte }\end{array}$ & Natural & $\begin{array}{l}\text { Carquin- } \\
\text { daño }\end{array}$ & $\begin{array}{c}\text { Sin } \\
\text { OCAS }\end{array}$ & $\begin{array}{l}\text { Tunga } \\
\text { Norte }\end{array}$ & Natural & $\begin{array}{l}\text { Carquin- } \\
\text { daño }\end{array}$ & $\begin{array}{c}\text { Sin } \\
\text { OCAS }\end{array}$ & $\begin{array}{l}\text { Tunga } \\
\text { Norte }\end{array}$ & Natural & $\begin{array}{l}\text { Carquin- } \\
\text { daño }\end{array}$ & $\begin{array}{c}\text { Sin } \\
\text { OCAS }\end{array}$ \\
\hline $\begin{array}{l}\text { Curaeus } \\
\text { curaeus }\end{array}$ & tordo & Ictiridae & $\mathrm{N}$ & & & & & 3 & & & & & 5 & 3 & \\
\hline $\begin{array}{l}\text { Colombina } \\
\text { picui }\end{array}$ & $\begin{array}{l}\text { tortolita } \\
\text { cuyana }\end{array}$ & Columbidae & $\mathrm{N}$ & & & & & & & & & & & & \\
\hline $\begin{array}{l}\text { Zenaida } \\
\text { auriculata }\end{array}$ & tórtola & Columbidae & $\mathrm{N}$ & 1 & & & & 3 & & & & 1 & & 1 & \\
\hline $\begin{array}{l}\text { Pteroptochos } \\
\text { megapodius }\end{array}$ & turca & Rhinocryptidae & En & & 1 & & & 3 & 2 & & & 1 & 2 & & \\
\hline $\begin{array}{l}\text { Colorhamphus } \\
\text { parvirostris }\end{array}$ & viudita & Tyrannidae & $\mathrm{N}$ & & & & & & & & & 1 & & & \\
\hline $\begin{array}{l}\text { Rhopospina } \\
\text { fruticeti }\end{array}$ & yal & Thraupidae & $\mathrm{N}$ & 2 & 2 & & & 1 & 7 & & & 1 & 2 & 1 & \\
\hline \multirow{2}{*}{\multicolumn{2}{|c|}{ TOTAL }} & \multicolumn{2}{|c|}{ Individuos } & 33 & 50 & 1 & 1 & 29 & 46 & 4 & 2 & 23 & 24 & 9 & 0 \\
\hline & & \multicolumn{2}{|c|}{ Especies } & 10 & 16 & 1 & 1 & 12 & 14 & 3 & 1 & 12 & 11 & 5 & 0 \\
\hline
\end{tabular}

\begin{tabular}{|c|c|c|c|c|c|c|c|c|c|c|c|c|c|c|c|}
\hline \multirow[b]{2}{*}{ Especie } & \multirow{2}{*}{$\begin{array}{l}\text { Nombre } \\
\text { común }\end{array}$} & \multirow[b]{2}{*}{ Familia } & \multirow[b]{2}{*}{ Origen } & \multicolumn{4}{|c|}{ Invierno 2020} & \multicolumn{4}{|c|}{ Primavera 2020} & \multicolumn{4}{|c|}{ Verano 2021} \\
\hline & & & & $\begin{array}{l}\text { Tunga } \\
\text { Norte } \\
\end{array}$ & Natural & $\begin{array}{l}\text { Carquin- } \\
\text { daño }\end{array}$ & $\begin{array}{c}\text { Sin } \\
\text { OCAS }\end{array}$ & $\begin{array}{l}\text { Tunga } \\
\text { Norte }\end{array}$ & Natural & $\begin{array}{l}\text { Carquin- } \\
\text { daño }\end{array}$ & $\begin{array}{c}\text { Sin } \\
\text { OCAS }\end{array}$ & $\begin{array}{l}\text { Tunga } \\
\text { Norte } \\
\end{array}$ & Natural & $\begin{array}{l}\text { Carquin- } \\
\text { daño }\end{array}$ & $\begin{array}{l}\text { Sin } \\
\text { OCAS }\end{array}$ \\
\hline $\begin{array}{l}\text { Anairetes } \\
\text { parulus }\end{array}$ & cachudito & Tyrannidae & $\mathrm{N}$ & 3 & 1 & & & 2 & 7 & & & 2 & 2 & & \\
\hline $\begin{array}{l}\text { Pseudasthenes } \\
\text { humicola }\end{array}$ & canastero & Furnariidae & En & & & & & & 1 & & & & 1 & & \\
\hline $\begin{array}{l}\text { Asthenes } \\
\text { modesta }\end{array}$ & $\begin{array}{l}\text { canastero } \\
\text { chico }\end{array}$ & Furnariidae & $\mathrm{N}$ & & & & & & & & & & & & \\
\hline $\begin{array}{l}\text { Dryobates } \\
\text { lignarius }\end{array}$ & carpinterito & Picidae & $\mathrm{N}$ & & & & & & 1 & & & & & & \\
\hline $\begin{array}{l}\text { Falco } \\
\text { sparverius }\end{array}$ & cernícalo & Falconidae & $\mathrm{N}$ & & & & & & 1 & & & & & & \\
\hline $\begin{array}{l}\text { Troglodytes } \\
\text { aedon }\end{array}$ & chercán & Troglodytidae & $\mathrm{N}$ & & & & & & 2 & & & 1 & 1 & & \\
\hline $\begin{array}{l}\text { Zonotrichia } \\
\text { capensis }\end{array}$ & chincol & Emberizidae & $\mathrm{N}$ & 3 & & 1 & 2 & 5 & 10 & 2 & 3 & 3 & & & 2 \\
\hline Sicalis luteola & chirihue & Thraupidae & $\mathrm{N}$ & 2 & & & & 2 & & & & & & & \\
\hline $\begin{array}{l}\text { Glaucidium } \\
\text { nana }\end{array}$ & chuncho & Strigidae & $\mathrm{N}$ & & 1 & & & 1 & & & & & & & \\
\hline $\begin{array}{l}\text { Callipepla } \\
\text { californica }\end{array}$ & codorniz & Odontophoridae & Ex & & 3 & & & & 1 & & & & & & \\
\hline Phrygilus gayi & $\begin{array}{l}\text { cometocino } \\
\text { de gay }\end{array}$ & Thraupidae & $\mathrm{N}$ & & 1 & 2 & & & 1 & 2 & & & & & \\
\hline Diuca diuca & diuca & Thraupidae & $\mathrm{N}$ & 3 & 1 & 2 & 1 & 3 & 3 & 1 & 2 & 3 & 1 & 1 & \\
\hline Pyrope pyrope & diucón & Tyrannidae & $\mathrm{N}$ & & & & & 2 & & & & 1 & 1 & & \\
\hline
\end{tabular}




\begin{tabular}{|c|c|c|c|c|c|c|c|c|c|c|c|c|c|c|c|}
\hline \multirow[b]{2}{*}{ Especie } & \multirow{2}{*}{$\begin{array}{l}\text { Nombre } \\
\text { común }\end{array}$} & \multirow[b]{2}{*}{ Familia } & \multirow[b]{2}{*}{ Origen } & \multicolumn{4}{|c|}{ Invierno 2020} & \multicolumn{4}{|c|}{$\begin{array}{l}\text { Primavera } 2020 \\
\end{array}$} & \multicolumn{4}{|c|}{ Verano 2021} \\
\hline & & & & $\begin{array}{l}\text { Tunga } \\
\text { Norte }\end{array}$ & Natural & $\begin{array}{l}\text { Carquin- } \\
\text { daño }\end{array}$ & $\begin{array}{c}\text { Sin } \\
\text { OCAS }\end{array}$ & $\begin{array}{l}\text { Tunga } \\
\text { Norte }\end{array}$ & Natural & $\begin{array}{l}\text { Carquin- } \\
\text { daño }\end{array}$ & $\begin{array}{c}\text { Sin } \\
\text { OCAS }\end{array}$ & $\begin{array}{l}\text { Tunga } \\
\text { Norte }\end{array}$ & Natural & $\begin{array}{l}\text { Carquin- } \\
\text { daño }\end{array}$ & $\begin{array}{c}\text { Sin } \\
\text { OCAS }\end{array}$ \\
\hline $\begin{array}{l}\text { Spinus } \\
\text { barbatus }\end{array}$ & jilguero & Fringillidae & $\mathrm{N}$ & & & & & 1 & & & & & & & \\
\hline Leistes loyca & loica & Ictiridae & $\mathrm{N}$ & & 1 & & & 3 & 1 & & & 2 & 1 & & \\
\hline Agriornis lividus & mero & Tyrannidae & En & & & & & & 1 & & & & & & \\
\hline $\begin{array}{l}\text { Sephanoides } \\
\text { sephaniodes }\end{array}$ & $\begin{array}{l}\text { picaflor } \\
\text { chico }\end{array}$ & Trochillidae & $\mathrm{N}$ & & 1 & & & 3 & 4 & & & & & & \\
\hline Patagona gigas & $\begin{array}{l}\text { picaflor } \\
\text { grande }\end{array}$ & Trochillidae & $\mathrm{N}$ & & & & & 2 & 5 & & & 4 & 3 & & \\
\hline Colaptes pitius & pitio & Picidae & $\mathrm{N}$ & & & & & & 1 & & & & & & \\
\hline $\begin{array}{l}\text { Scelorchilius } \\
\text { albicollis }\end{array}$ & tapaculo & Rhinocryptidae & En & & 1 & & & & 2 & & & 1 & 2 & & \\
\hline Mimus thenca & tenca & Mimidae & En & 3 & 3 & & & 4 & 5 & 1 & & 4 & 3 & 1 & \\
\hline $\begin{array}{l}\text { Leptasthenura } \\
\text { aegithaloides }\end{array}$ & tijeral & Furnariidae & $\mathrm{N}$ & & & & & 1 & 2 & & & 1 & & & \\
\hline $\begin{array}{l}\text { Milvago } \\
\text { chimango }\end{array}$ & tiuque & Falconidae & $\mathrm{N}$ & & & & & & 3 & & & & & & \\
\hline $\begin{array}{l}\text { Curaeus } \\
\text { curaeus }\end{array}$ & tordo & Ictiridae & $\mathrm{N}$ & & 4 & & & 3 & 4 & & & 4 & 3 & 1 & \\
\hline $\begin{array}{l}\text { Colombina } \\
\text { picui }\end{array}$ & $\begin{array}{l}\text { tortolita } \\
\text { cuyana }\end{array}$ & Columbidae & $\mathrm{N}$ & & & & & & & & & & 1 & & \\
\hline $\begin{array}{l}\text { Zenaida } \\
\text { auriculata }\end{array}$ & tórtola & Columbidae & $\mathrm{N}$ & 1 & & & & 3 & & & & & & & \\
\hline $\begin{array}{l}\text { Pteroptochos } \\
\text { megapodius }\end{array}$ & turca & Rhinocryptidae & En & 1 & 1 & & & 1 & 1 & & & & & & \\
\hline $\begin{array}{l}\text { Colorhamphus } \\
\text { parvirostris }\end{array}$ & viudita & Tyrannidae & $\mathrm{N}$ & & & & & & & & & & & & \\
\hline $\begin{array}{l}\text { Rhopospina } \\
\text { fruticeti }\end{array}$ & yal & Thraupidae & $\mathrm{N}$ & & 2 & & & 4 & 7 & & & 1 & & & 1 \\
\hline & \multicolumn{2}{|c|}{ Individuos } & 16 & 20 & 5 & 3 & 40 & 63 & 6 & 5 & 27 & 19 & 3 & 3 \\
\hline \multicolumn{2}{|c|}{ TOTAL } & \multicolumn{2}{|c|}{ Especies } & 7 & 12 & 3 & 2 & 16 & 21 & 4 & 2 & 12 & 11 & 3 & 2 \\
\hline
\end{tabular}


Cuadro 4. Riqueza de Especies Detectadas Según Área de Estudio.

\begin{tabular}{|c|c|c|c|c|c|c|c|c|}
\hline \multirow{2}{*}{$\begin{array}{l}\text { Estaciones } \\
\text { calendario }\end{array}$} & \multicolumn{4}{|c|}{ Artrópodos (orden) } & \multicolumn{4}{|c|}{ Reptiles (sp) } \\
\hline & $\begin{array}{l}\text { Tunga } \\
\text { Norte }\end{array}$ & Natural & Carquindaño & Sin OCAS & Tunga Norte & Natural & Carquindaño & Sin OCAS \\
\hline Primavera 2019 & 6 & 5 & 1 & 1 & 1 & 1 & 0 & 0 \\
\hline Verano 2020 & 5 & 7 & 3 & 0 & 1 & 1 & 0 & 0 \\
\hline Otoño 2020 & 5 & 5 & 3 & 2 & 1 & 1 & 0 & 0 \\
\hline Invierno 2020 & 5 & 5 & 3 & 2 & 1 & 1 & 0 & 0 \\
\hline Primavera 2020 & 6 & 7 & 3 & 3 & 1 & 1 & 0 & 0 \\
\hline Verano 2021 & 7 & 5 & 3 & 3 & 1 & 1 & 0 & 0 \\
\hline \multirow{2}{*}{$\begin{array}{l}\text { Estaciones } \\
\text { calendario }\end{array}$} & \multicolumn{4}{|c|}{ Aves (sp) } & \multicolumn{4}{|c|}{ Mamíferos (sp) } \\
\hline & $\begin{array}{l}\text { Tunga } \\
\text { Norte }\end{array}$ & Natural & Carquindaño & Sin OCAS & Tunga Norte & Natural & Carquindaño & Sin OCAS \\
\hline Primavera 2019 & 10 & 16 & 1 & 1 & 1 & 0 & 0 & 0 \\
\hline Verano 2020 & 12 & 13 & 3 & 1 & 2 & 0 & 0 & 0 \\
\hline Otoño 2020 & 12 & 13 & 5 & 0 & 2 & 0 & 0 & 0 \\
\hline Invierno 2020 & 7 & 12 & 3 & 2 & 2 & 0 & 0 & 0 \\
\hline Primavera 2020 & 16 & 21 & 4 & 2 & 2 & 0 & 0 & 0 \\
\hline Verano 2021 & 12 & 11 & 3 & 2 & 2 & 1 & 0 & 0 \\
\hline
\end{tabular}

El cálculo de los índices de Shannon-Wiener (Diversidad Alfa) y Jaccard (Diversidad Beta) solo se estimó para los datos de aves, esto debido a que el $n$ de la muestra $(n=29)$, así lo permite. El índice de ShannonWiener fluctuó entre 1 y 2,9, indicando que las áreas con mayor diversidad de aves y las que están representadas casi por el mismo número de especies son Tunga Norte y Natural, siendo el valor más bajo para el área Sin OCAS. Los valores obtenidos (Cuadro $N^{\circ} 5$ ) muestran que el gradiente de mayor a menor diversidad observado corresponde a Natural (25 especies)>Tunga Norte (20 especies)>Carquindaño (9 especies)>Sin OCAS (4 especies). El valor de diversidad alfa en la mayoría de los ecosistemas naturales varía entre 0,5 y 5, aunque su valor normal está entre 2 y 3 ; valores inferiores a 2 se consideran bajos en diversidad y superiores a 3 son altos en diversidad de especies (Magurran, 1988; Pla, 2006; ChávezVillavicencio, 2017). En este caso los valores para Tunga Norte y Natural son cercanos al valor 3, por lo tanto, la diversidad de aves para Tunga Norte y Natural es alta.

Cuadro 5. Indice de Shannon-Weaver (Diversidad Alfa) para Áreas Estudiadas.

\begin{tabular}{lcc}
\hline Área Estudiada & $\begin{array}{c}\text { Diversidad } \\
\text { Alfa }\end{array}$ & $\begin{array}{c}\text { Total Especies } \\
\text { Aves }\end{array}$ \\
\hline Tunga Norte & 2,6 & 20 \\
\hline Natural & 2,9 & 25 \\
\hline Carquindaño & 2 & 9 \\
\hline Sin OCAS & 1 & 4 \\
\hline
\end{tabular}

El mayor valor de similitud registrado con el índice Beta fue entre las áreas Tunga Norte y Natural con un $55,2 \%$ de similitud y con 16 especies de aves compartidas, seguido por Natural y Carquindaño con $32 \%$ y 8 especies compartidas. El área Sin OCAS no fue considera para el cálculo debido al bajo número de especies de aves presentes en este caso (Cuadro 6).

Cuadro 6. Índice de Jaccard (Diversidad Beta) Según Área Estudiada.

\begin{tabular}{lcc}
\hline Localidad & $\begin{array}{c}\text { Diversidad } \\
\text { Beta (\%) }\end{array}$ & $\begin{array}{c}\text { Total Especies } \\
\text { Compartidas }\end{array}$ \\
\hline Tunga Norte/Natural & 55,2 & 16 \\
\hline Natural/Carquindaño & 32 & 8 \\
\hline Tunga Norte/Carquindaño & 31 & 7 \\
\hline
\end{tabular}


En cuanto a la abundancia, se observó un patrón similar al encontrado para la riqueza específica. Las áreas de ambiente Natural y Con OCAS de más de 20 años muestran valores significativamente mayores que en Carquindaño y Sin OCAS $\left(F_{(3,20)}=4,18 ; P=0,02\right)$. Los mayores valores para los artrópodos se detectaron en otoño 2020 (159 y 163 artrópodos/trampas noche, respectivamente), en cambio dichos valores para las áreas Carquindaño y Sin OCAS fueron 47 y 31 individuos, respectivamente. La caída observada en invierno se puede explicar principalmente por el frío, ya que los artrópodos son organismos ectotermos, por lo tanto, su actividad está directamente relacionada con la temperatura del ambiente (Bozinovic y Cavieres, 2019). La abundancia observada en las áreas Tunga Norte y Natural fueron significativamente mayores que lo detectado en Carquindaño y Sin OCAS $\left(F_{(3,20)}=22,51 ; P<0,001\right)$. Los mayores valores correspondieron al área Natural (primavera 2020) y Tunga Norte (primavera 2020), alcanzando los 32 y 20 individuos/ha, respectivamente. Tanto los reptiles como los mamíferos estuvieron ausentes en las áreas Carquindaño y sin OCAS (Cuadro 7, Figura 3).

Cuadro 7. Abundancia ( $\mathrm{N}^{\circ}$ de Individuos) Detectada en las Áreas de Estudio.

\begin{tabular}{|c|c|c|c|c|c|c|c|c|}
\hline \multirow{2}{*}{$\begin{array}{l}\text { Estaciones } \\
\text { Calendario }\end{array}$} & \multicolumn{4}{|c|}{ Artrópodos/Trampas Noche } & \multicolumn{4}{|c|}{ Reptiles } \\
\hline & $\begin{array}{l}\text { Tunga } \\
\text { Norte }\end{array}$ & Natural & Carquindaño & Sin OCAS & Tunga Norte & Natural & Carquindaño & Sin OCAS \\
\hline Primavera 2019 & 34 & 40 & 1 & 1 & 2 & 3 & 0 & 0 \\
\hline Verano 2020 & 73 & 86 & 14 & 0 & 3 & 5 & 0 & 0 \\
\hline Otoño 2020 & 163 & 159 & 47 & 31 & 5 & 5 & 0 & 0 \\
\hline Invierno 2020 & 17 & 17 & 12 & 9 & 3 & 4 & 0 & 0 \\
\hline Primavera 2020 & 54 & 58 & 18 & 21 & 4 & 6 & 0 & 0 \\
\hline Verano 2021 & 91 & 56 & 26 & 24 & 6 & 6 & 0 & 0 \\
\hline \multirow{2}{*}{$\begin{array}{l}\text { Estaciones } \\
\text { Calendario }\end{array}$} & \multicolumn{4}{|c|}{ Aves/ha } & \multicolumn{4}{|c|}{ Mamíferos } \\
\hline & $\begin{array}{l}\text { Tunga } \\
\text { Norte }\end{array}$ & Natural & Carquindaño & Sin OCAS & Tunga Norte & Natural & Carquindaño & Sin OCAS \\
\hline Primavera 2019 & 17 & 17 & 1 & 1 & 1 & 0 & 0 & 0 \\
\hline Verano 2020 & 15 & 21 & 2 & 1 & 2 & 0 & 0 & 0 \\
\hline Otoño 2020 & 12 & 15 & 5 & 0 & 2 & 0 & 0 & 0 \\
\hline Invierno 2020 & 8 & 10 & 3 & 2 & 2 & 0 & 0 & 0 \\
\hline Primavera 2020 & 20 & 32 & 3 & 3 & 2 & 0 & 0 & 0 \\
\hline Verano 2021 & 14 & 16 & 2 & 2 & 2 & 1 & 0 & 0 \\
\hline
\end{tabular}

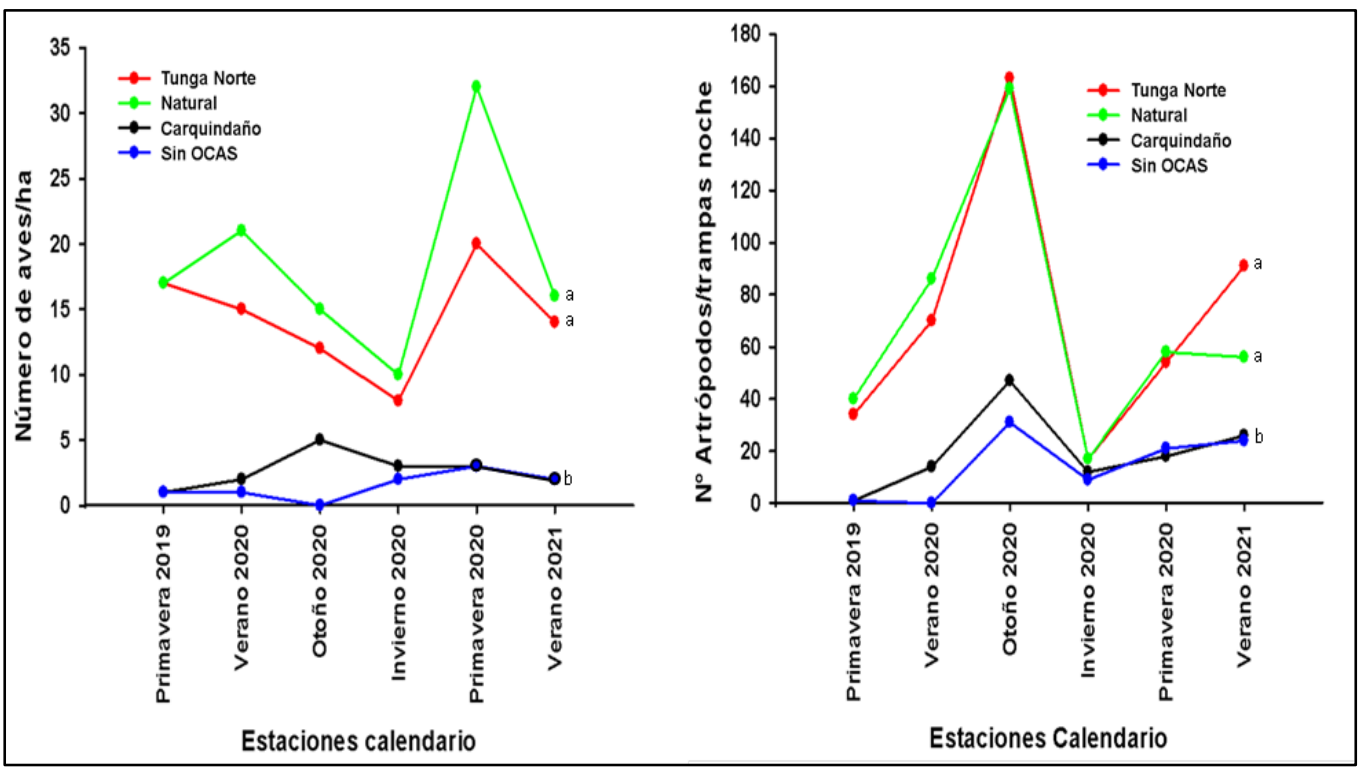

Figura 3. Abundancia por Grupo Taxonómico en las Áreas de Estudio. (Letras distintas indican diferencias significativas ( $p<0.05$; prueba de Tukey; nivel de confianza 95\%) 


\section{DISCUSIÓN Y CONCLUSIONES}

La actual degradación y el alto grado de perturbación de los ecosistemas naturales hace necesario buscar e idear diferentes métodos para mitigar o reparar en lo posible el daño provocado al ambiente. Desde esta necesidad surgen las obras de conservación de agua y suelo (OCAS) con vegetación, obras que permiten fundamentalmente cosechar aguas lluvias que se acumula en el suelo e infiltra hacia las napas subterráneas, constituyéndose en un aporte hídrico para la vegetación que allí se establezca y la sociedad, hecho que mitiga la degradación de los suelos y el deterioro de los ecosistemas. Es por estos motivos que las OCAS con vegetación son una herramienta tecnológica que ayuda a la adaptación al cambio climático y a la lucha contra la desertificación (INFOR, 2018). La calidad de los fragmentos de hábitat, en cuanto a la composición y riqueza vegetacional en sí misma puede afectar a la supervivencia a largo plazo de especies animales. Así, la presencia de recursos críticos como es la disponibilidad de alimento y refugio en forma de oquedades en la vegetación para ciertas especies de aves, es también un factor que los gestores deben tener en cuenta en la aplicación de estrategias de conservación y/o restauración, por ejemplo, Oasificación mediante OCAS (Herrera, 2011).

Los resultados preliminares de este trabajo muestran que las áreas en estudio presentan diferencias en sus características vegetacionales y de cobertura, hecho que se traduciría en una mayor complejidad estructural del hábitat en Tunga Norte y Natural (70-60\% cobertura promedio, respectivamente), siendo la cobertura vegetal un factor importante que podría estar influenciando la biodiversidad animal existente. Por el contrario, las áreas más perturbadas, Carquindaño y Sin OCAS (8-1\% cobertura promedio, respectivamente), no son hábitat con características vegetacionales determinantes para la existencia de biodiversidad animal. Adicionalmente, el índice alfa indica un gradiente de riqueza que se corresponde con la degradación de las áreas estudiadas, ambientes con mayor cobertura vegetal presentan valores similares de diversidad de aves. Al cabo de 23 años, el área de Tunga Norte alcanza un 56\% de similitud con el ambiente Natural sin intervención.

Lo anterior apoya la hipótesis de que las características estructurales del medio son de primordial importancia en la determinación de la distribución y niveles poblacionales de las especies de fauna. La influencia de la estructura de la vegetación debe producirse fundamentalmente a nivel de la nidificación (emplazamiento de los nidos), protección antidepredación y áreas de alimentación (Antogiovanni y Metzger, 2005; Garcia, 2011; Herrera, 2011, Herrera et al., 2011). La presencia en Tunga Norte del roedor fosorial Spalacopus cyanus refuerza este planteamiento, ya que este mamífero prefiere hábitat abiertos, bien drenados, de suelo blando y con gran cubierta vegetal de matorrales y praderas por sobre el $60 \%$ (RootBernsteina y Jaklsic, 2015).

Desde esta perspectiva, las OCAS establecidas por INFOR incorporan técnicas de manejo hidrológicoforestal orientadas a disminuir la escorrentía y favorecer la infiltración, junto con la incorporación de especies vegetales arbóreas y arbustivas. Pero es un proceso que no solo se focaliza en la conservación de agua y suelo, sino que contribuye a la oasificación y conectividad de estas áreas degradadas al incorporar vegetación, permitiendo recuperar algunos servicios ecosistémicos, tal como la biodiversidad. La vegetación de las zonas áridas de Chile juega un rol fundamental en la persistencia y funcionamiento de los ecosistemas mediterráneos, siendo un factor clave para el funcionamiento hídrico y la mantención de la biodiversidad, contribuyendo a frenar el desgaste del relieve y la erosión causada por el viento y también puede interceptar neblina costera proporcionando humedad en las capas superficiales del suelo (Gutiérrez y Squeo, 2004).

La conectividad generada por medio de la osificación es considerada como un elemento vital de la estructura del paisaje debido a su importancia para la supervivencia de especies (D'Eon et al., 2002) y es definida como el grado en el cual un paisaje facilita o impide el movimiento de organismos entre diferentes parches de hábitat (Tischendorf y Fahrig, 2000). Bajo este contexto, los corredores biológicos mediante la oasificación son reconocidos por su importancia como conectores de elementos y funciones ecológicas, cuyo fin es proporcionar conectividad entre paisajes, ecosistemas y hábitats, naturales o modificados, para asegurar el mantenimiento de la biodiversidad y los procesos ecológicos-evolutivos (Oviedo y Gutiérrez, 2016). 
En este sentido, las OCAS son una herramienta tecnológica eficaz, donde el patrón de biodiversidad observado hasta el momento permite concluir que las OCAS de más de 20 años han alcanzado en términos de biodiversidad de aves un $56 \%$ de similitud con lo observado en lugares naturales no intervenidos. Así, las OCAS con vegetación junto con acumular agua y conservar suelo, contribuirían a la osificación de estas áreas degradadas, traduciéndose en una recuperación importante de la biodiversidad de aves y artrópodos presente en estas áreas, permitiendo concomitantemente disminuir la fragmentación y permitiendo la conectividad biológica (corredores biológicos) con otras áreas naturales. De esta manera, la biodiversidad podría ser utilizada como un indicador de recuperación y calidad de hábitat de estos ambientes degradados.

Aun cuando los resultados presentados muestran una tendencia positiva en la recuperación de hábitats para fauna, estos son preliminares y se requiere considerar periodos de entre 5-10 años, para validar y robustecer los resultados.

\section{RECONOCIMIENTOS}

La presente publicación entrega los resultados preliminares del programa de investigación "Monitoreo de obras de conservación de agua y suelo (OCAS) en Cuencas de Ecosistemas Áridos y Semiáridos del Centro -Norte de Chile", ejecutado por el Instituto Forestal (INFOR) durante los años 2019-2021, con el apoyo del Ministerio de Agricultura (MINAGRI) y en colaboración con Comunidades Agrícolas y propietarios.

\section{REFERENCIAS}

AGRIMED. (2017). Atlas Agroclimático de Chile, Tomo II. Estado actual y tendencias del clima. Universidad de Chile, Santiago.

Antogiovanni, M. \& Metzger, J.P. (2005). Influence of matrix habitat on the occurrence of insectivorous bird species in Amazonian forest fragments. Biological Conservation, 122. Pp: 441-451. https://doi.org/10.1016/j.biocon.2004.09.005

Arim, P., Marquet, P.A. \& Jaksic F.M. (2007. On the Relationship between Productivity and Food Chain Length at Different Ecological. The American Naturalist, 169 (1): 62-72. https://doi.org/10.1086/510210

Awade, M. y Metzger, J.P. (2008). Using gap-crossing capacity to evaluate functional connectivity of two Atlantic rainforest birds and their response to fragmentation. Austral Ecology, 33. Pp: 863-871. https://doi.org/10.1111/j.1442-9993.2008.01857.x

Bennett, A.F. 1998. Linkages in the Landscape: The Role of Corridors and Connectivity in Wildlife Conservation. Gland, Switzerland, IUCN. 254p.

Bennett, A. (2003). Linkages in the landscape: the role of corridors and connectivity in wildlife conservation. Gland, Switzerland, IUCN. 254 p. EN: Donoso, C., González, M. y Lara, A. 2014. Ecología Forestal. Bases para el Manejo Sustentable y Conservación de los Bosque Mediterráneos de Chile. Pp: 583-605. https://doi.org/10.2305/IUCN.CH.2004.FR.1.en

Blondel J., Ferry, C. \& Frochot, B. (1981). Point counts with unlimited distance. Studies in Avian Biology, 6. Pp: 414420.

Bozinovic, F. \& Cavieres, L.A. (2019). La vulnerabilidad de los organismos al cambio climático: rol de la fisiología y la adaptación. Ediciones CAPES-UC. Santiago.

Bustamante R. \& Grez A. (1995). Consecuencias ecológicas de la fragmentación de los bosques nativos. Ciencia y ambiente, 11(2): 58-63.

CIREN. (2010). Determinación de la erosión actual y potencial de los suelos de Chile: Región de Coquimbo. Síntesis de resultados. Disponible en: http://bibliotecadigital.ciren.cl/handle/123456789/2059. Consulta: abril, 2021.

CIREN. (2012). Estado actual de los suelos de la Región de Coquimbo. Uso y degradación. Disponible en: http://bibliotecadigital.ciren.cl/bitstream/handle/123456789/2033/PC17613. pdf?sequence=1\&isAllowed=y. Consulta: abril, 2021.

Chávez-Villavicencio, L. (2017). Diversidad alfa y beta de las aves terrestres en ecosistemas no urbanizado y urbanizado de La Herradura (Coquimbo - Chile). The Biologist (Lima), 15. Pp: 329-336. https://doi.org/10.24039/rtb2017152192 
CONAF. (2016). Diagnóstico de la desertificación en Chile y sus efectos en el desarrollo Sustentable. Disponible en: https://www.researchgate.net/publication/312139716_Diagnostico_de_la_desertificacion_en_Chile_y_sus_ef ectos_en_el_desarrollo_sustentable. Consulta: abril, 2021.

CONAF. (2021). Proyecto Manejo Sustentable de la Tierra Área Piloto Combarbalá: Corredor de Conservación Reserva Nacional Las Chinchillas - Derecho Real de Conservación Hacienda el Durazno. Proyecto Manejo Sustentable de la Tierra (PMST) perteneciente a la Estrategia Nacional de Cambio Climático y Recursos

D'Eon, R.G., Parfitt, G.I. \& Fortin, M.J. (2002). Landscape connectivity as a function of scale and organism vagility in a real forested lanscape. Conservation Ecology, 6(2): art. 10. https://doi.org/10.5751/ES-00436-060210

DGA. (2021). Decretos de Escasez Hídrica. https://dga.mop.gob.cl/noticias/Paginas/DetalledeNoticias.aspx?item=745. Visitada en abril 2021.

Flores, J. P., Espinoza, M., Martinez, E., Henríquez, G., Avendaño, P., Torres, P. \& Ahumada, I. (2010). Determinación de la erosión actual de los suelos de Chile. Centro de Información de Recursos Naturales (CIREN), Santiago.

Gacitúa, S., González, M., Hernández, J., Villalobos, E. \& Montenegro, J. (2020). Diseño e implementación de modelos agroforestales de secano en obras de conservación de agua y suelos: Región de Coquimbo. Manual N 54. Instituto Forestal.

Gajardo, R. (1994). La vegetación natural de Chile. Clasificación y distribución geográfica. Editorial Universitaria, Santiago.

García, D. (2011). Efectos biológicos de la fragmentación de hábitats: nuevas aproximaciones para resolver un viejo problema. Ecosistemas, 20. Pp: 1-11.

González, M., Salinas, J. \& Montenegro, J. (2020). Sistemas Silvopastorales en Chile. Generalidades y Ejemplos. Manual $\mathrm{N}^{\circ} 58$, Instituto Forestal.

GORE Coquimbo. (2010). Descripción y caracterización de la Comuna de Canela y sus Comunidades Agrícolas, Región de Coquimbo. En: https://www.gorecoquimbo.cl/gorecoquimbo/site/artic/20160425/asocfile/20160425131438/cos_aguas_lluvia S_tomo_i_anexo_4.pdf. Consulta: abril, 2021.

GORE Coquimbo. (2016). Anexo: Descripción y caracterización de la comuna de Canela y sus comunidades agrícolas, Región de Coquimbo. En: https://www.gorecoquimbo.cl > site > cos_aguas_lluvias_tomo_i_anexo_4. Consulta: junio, 2020.

GORE Coquimbo. (2017). Congreso del Agua: Desafíos para la Macro zona Norte. En: https://www.gorecoquimbo.cl/congreso-de-agua-2017/gorecoquimbo/2017-03-20/103050.html. Consulta: abril, 2021.

Gutiérrez, J.R. \& Squeo, F.A. (2004). Importancia de los arbustos leñosos en los ecosistemas semiáridos de Chile. Ecosistemas, 13. Pp: 36-45.

Herrera, J.M. (2011). El papel de la matriz en el mantenimiento de la biodiversidad en hábitats fragmentados. De la teoría ecológica al desarrollo de estrategias de conservación. Ecosistemas, 20: 21-34.

Herrera, J.M., García, D., Martínez, D. \& Valdés, A. (2011). Regional vs. local effects of habitat loss and fragmentation on two plant-animal interactions. Ecography, 34. Pp: 606-615. https://doi.org/10.1111/j.16000587.2010.06521.x

Iriarte, A. (2008). Mamíferos de Chile. Lynx Ediciones. Barcelona, España. 420 p.

Jiménez, J.E. (2000). Effect of sample size, plot size, and counting time on estimates of avian diversity and abundance in a Chilean rainforest. Journal of Field Ornithology, 71. Pp: 66-87. https://doi.org/10.1648/0273-8570-71.1.66

Jorquera, C. (2001). Evolución Agropecuaria de la Región de Coquimbo: Análisis Contextual para la Conservación de la Vegetación Nativa. En: Squeo, F.A., Arancio, G. \& Gutiérrez, J.R. (Eds). Libro Rojo de la Flora Nativa y de los Sitios Prioritarios para su Conservación: Región de Coquimbo. Ediciones Universidad de La Serena. Pp: 225-237).

Labra, F., González, M., Gacitúa, S., Montenegro, J., Villalobos, E. \& Gómez, A. (2018). Manual para la implementación de obras de conservación de suelos y cosecha de aguas lluvias en Alhué. Manual $\mathrm{N}^{\circ} 49$, Instituto Forestal.

Larraín, S., Aedo, M.P., Navarrete, K. \& Villarroel, C. (2010). Marco Jurídico para la gestión del agua en Chile: Diagnóstico y desafíos.

En: https://sswm.info/sites/default/files/reference_attachments/LARRAIN\%20S\%20et\%20al\%202010\%20M\%20J uridico\%20Gestion\%20Agua\%20Chile-SPAN̄ISH.pdf. Consulta: abril, 2021. 
Luebert, F. \& Pliscoff, P. (2006). Sinopsis bioclimática y vegetacional de Chile. Editorial Universitaria. Santiago.

Magurran, A. E. (1988). Ecological Diversity and its Measurement. Princeton University Press. New Jersey, USA. 192 p. https://doi.org/10.1007/978-94-015-7358-0

Magurran, A. E. (2004). Measuring biological diversity. Blackwell Publishing. Oxford, UK. 256 p.

Martínez de Azagra, A., Mongil, J., del Río San José, J. \& Rojo, I. (2006). Conceptos, modelos y ejemplos sobre oasificación. Ecología, 20. Pp: 453-469.

McRae B. H., Hall S.A., Beier P. \& Theobald D.M. (2012). Where to Restore Ecological Connectivity? Detecting Barriers and Quantifying Restoration Benefits. PLoS ONE, https://doi.org/10.1371/journal.pone.0052604

Mella, J.E. (2005). Guía de campo Reptiles de Chile, zona central. Centro de Ecología Aplicada CEA. Santiago.

Mongil, J. \& Martínez de Azagra, A. (2006). Diseño de repoblaciones forestales en zonas áridas: tamaño del microembalse y relación entre el área de impluvio y el área de recepción. Investigaciones Geográficas, 40. Pp: 201-226. https://doi.org/10.14198/INGEO2006.40.10

Morales, C., Acevedo, J., Araníbar, Z. \& Dascal, G. (2016). Chile: Los costos de inacción de la desertificación y degradación de las tierras. Resultados de un estudio del Programa de las Naciones Unidas para el Desarrollo. Santiago. 112 p.

Muñoz-Pedreros, A. \& Yáñez J. (2000). Mamíferos de Chile. Centro de Estudios Agrarios y Ambientales (CEA) Ediciones. Valdivia, Chile. viii +464 p.

Núñez, H. \& Jaksic, F.M. (1992). Lista comentada de los reptiles terrestres de Chile continental. Boletín del Museo Nacional de Historia Natural (Chile), 43. Pp: 63-91.

Oviedo J. P. \& Gutiérrez M.L. (2016). El Problema de la Falta de Conectividad entre Áreas Silvestres en Chile: El caso de la Cuenca del Río Malleco. Memoria para optar al grado de Licenciado en Ciencias Jurídicas y Sociales. Universidad de Chile, Departamento de Enseñanza Clínica del Derecho. 101 p.

Perret, S.; Gacitúa, S. \& Montenegro, J. (2011). Técnicas de cosecha de aguas lluvia y conservación de suelos para la oasificación del norte Chileno. Instituto Forestal. Manual $N^{\circ} 44 . \quad$ En: https://bibliotecadigital.infor.cl/handle/20.500.12. Consulta: abril, 2021.

Pincheira, D. \& Núñez, H. (2005). Las especies chilenas del genero Liolaemus Weigmaner, 1834 (Iguana: Tropiduridae: Liolaeminae). Taxonomía, Sistemática y Evolución. Museo de Historia Natural: Publicación Ocasional № 59.

Pla, L. (2006). Biodiversidad: inferencia basada en el índice de Shannon y la riqueza. Interciencia, 31. Pp: 583-590.

Rappaport, D., Tambosi, I. \& Metzger, J. (2015). A landscape triage approach: combining spatial and temporal dynamics to prioritize restoration and conservation. Journal of Applied Ecology, 52. Pp: $590-601$. https://doi.org/10.1111/1365-2664.12405

Reynolds, R.T., Scott, J.M. \& Nussbaum, R.A. (1980). Avariable circular-plot method for estimating bird numbers. Condor, 82. Pp: 309-313. https://doi.org/10.2307/1367399

Root-Bernsteina, M. \& Jaksic F.M. (2015). Ecosystem process interactions betweencentral Chilean hábitats. Global Ecology and Conservation, 3. Pp: 776-788. https://doi.org/10.1016/i.gecco.2015.04.007

Sarmiento, L., Smith, J.K. \& Monasterio, M. (2002). Balancing Conservation of Biodiversity and Economical Profit in the Agriculture of the High Venezuelan Andes: Are Fallow Systems an Alternative? In: Korner, Ch. \& Spehn, E.M. (Eds). Mountain Biodiversity- A global assessment. Parthenon Publisher. Pp: 285-295,

Tambosi, L., Martensen, A., Ribeiro M. \& Metzger, J. (2014). A Framework to Optimize Biodiversity Restoration Efforts Based on Habitat Amount and Landscape Connectivity. Restoration Ecology, 22. Pp: 169-177. https://doi.org/10.1111/rec.12049

Tischendorf L. \& Fahrig L. (2000). On the usage and measurement of landscape connectivity. Oikos, 90. Pp: 7-19. https://doi.org/10.1034/j.1600-0706.2000.900102.X

Vásquez, R.A. \& Simonetti J.A. (1999). Life history traits and sensitivity to landscape change: the case of birds and mammals of mediterranean Chile. Revista Chilena de Historia Natural, 72. Pp: 517-525.

Veloso, A. \& Navarro, J. (1988). Lista sistemática y distribución geográfica de anfibios y reptiles de Chile. Bollettino del Museo Regionale di Scienze Naturali, Torino, 6. Pp: 481-539. 
Ciencia \& Investigación Forestal Vol. $27 \mathrm{~N}^{\circ} 2$. Agosto 2021

Willson M.F., de Santo, T.L., Sabag, C. \& Armesto, J.J. (1994). Avian communities of fragmented southtemperate rainforests in Chile. Conservation Biology, 8. Pp: 508-520. https://doi.org/10.1046/i.15231739.1994.08020508.x

https://revista.infor.cl 